\title{
Visualization of a Capsule Entry Vehicle Reaction-Control System (RCS) Thruster
}

\author{
P.M. Danehy ${ }^{*}$, J.A. Wilkes ${ }^{\dagger}$, G.J. Brauckmann ${ }^{\ddagger}$, D.W. Alderfer ${ }^{\S}$, S.B. Jones ${ }^{* *}$ \\ NASA Langley Research Center, Hampton VA, 23681-2199 \\ and \\ D. P. Patry ${ }^{\dagger \dagger}$ \\ Swales Aerospace, Hampton VA, 23681-2199
}

\begin{abstract}
Planar laser-induced fluorescence (PLIF) was used to visualize the reaction control system (RCS) jet flow emanating from the aft-body of an Apollo-geometry capsule test article in the NASA Langley Research Center 31-Inch Mach 10 Air wind tunnel. The RCS jet was oriented normal to the aft surface of the model and had a nominal Mach number of 2.94. The composition of the jet gas by mass was $95 \%$ nitrogen $\left(\mathrm{N}_{2}\right)$ and $5 \%$ nitric oxide (NO). The RCS jet flowrate varied between zero and 0.5 standard liters per minute and the angle of attack and tunnel stagnation pressure were also varied. PLIF was used to excite the NO molecules for flow visualization. These flow visualization images were processed to determine the trajectory and to quantify the flapping of the RCS jet. The spatial resolution of the jet trajectory measurement was about $1 \mathrm{~mm}$ and the single-shot precision of the measurement was estimated to be $0.02 \mathrm{~mm}$ in the far field of the jet plume. The jet flapping, measured by the standard deviation of the jet centerline position was as large as $0.9 \mathbf{~ m m}$, while the jet was $1.5-4 \mathrm{~mm}$ in diameter (full width at half maximum). Schlieren flow visualization images were obtained for comparison with the PLIF. Surface pressures were also measured and presented. Virtual Diagnostics Interface (ViDI) technology developed at NASA Langley was used to superimpose and visualize the data sets. The measurements demonstrate some of the capabilities of the PLIF method while providing a test case for computational fluid dynamics (CFD) validation.
\end{abstract}

\section{Introduction}

A capsule design following the heritage of Apollo was recently selected for NASA's Crew Exploration Vehicle (CEV). ${ }^{1}$ Capsules have some distinct advantages over lifting body shapes. They have a low structural weight (due to a low ratio of surface area to volume) and are less complex (typically no movable control surfaces). However, this reduced complexity comes at the expense of maneuverability. ${ }^{2}$ Their smaller size allows them to be launched into space on the most forward point of a launch stack - a safer configuration compared to attaching the vehicle to the side of a rocket. Reaction control system (RCS) jet thrusters offer the primary means for steering capsules both on-orbit and during planetary entry. RCS jets can be used to provide pitch, yaw, and roll control to change the direction of the capsule's lift vector during entry. This requirement is increasingly important for the CEV as pinpoint ground (at Earth and eventually planetary) landings are planned for the CEV program. Accurate understanding and modeling of RCS jets, their trajectories, and their influence on the flowfield and therefore the moments acting on the vehicle are critical for designing and predicting the performance of new capsule-based space vehicles. In this paper, we use planar laser-induced fluorescence (PLIF) to qualitatively and quantitatively visualize

\footnotetext{
${ }^{*}$ Research Scientist, Advanced Sensing and Optical Measurement Branch, MS 493, AIAA Associate Fellow.

${ }^{\dagger}$ PhD Student, Department of Physics, College of William and Mary, Williamsburg, VA and NASA Graduate Coop

Student, Advanced Sensing and Optical Measurement Branch, MS 493, AIAA Student Member.

${ }^{\ddagger}$ Test Engineer, Aerothermodynamics Branch, MS 408A, AIAA Member.

${ }^{\S}$ Research Scientist, Advanced Sensing and Optical Measurement Branch, MS 493

${ }^{* *}$ Optic and Electronic Technician, Advanced Sensing and Optical Measurement Branch, MS 493

${ }^{+\dagger}$ Information Technology Specialist, MS 493, NASA Langley Research Center.
} 
a RCS jet for an Apollo-shaped capsule. The primary goal of this experiment was to demonstrate the capabilities of PLIF for visualizing RCS jets and for performing quantitative measurements. However, this data set may serve as a comparison case for computational fluid dynamics (CFD) simulations.

While the CEV RCS strategy has not yet been clearly defined, it is useful to review the Apollo command module (CM) RCS to put the present work into context. The Apollo CM RCS was responsible for controlling the vehicle attitude from the point of separation from the service module until the parachutes were deployed prior to splashdown. The RCS was also designed to contribute in various crew abort scenarios. The Apollo RCS consisted of 12 maneuvering jets -4 each for pitch, roll and yaw, all of which exhausted on the leeward side of the capsule. ${ }^{3}$ The Apollo RCS jets were pressure fed and operated with hypergolic (self-igniting) propellants $\left(\mathrm{N}_{2} \mathrm{O}_{4}\right.$ and monomethyl hyrdrazine), and the engines were designed to each produce 93 pounds of thrust. ${ }^{3}$ The converging/diverging jet nozzle had a 7:1 expansion ratio, ${ }^{3}$ resulting in a nominal Mach number of 3.5 (assuming $\gamma$ $=1.4$ ). The pressure in the combustion chamber was 150 psia. $^{3}$ Engines typically fired hundreds of times during a single mission with burn times typically a fraction of a second but also lasting a minute or longer. ${ }^{3}$ The engine startup and shutdown times were on the order of $10 \mathrm{~ms}$, thus the startup and shutdown were short compared to typical firing durations. ${ }^{3}$

One of the major goals in testing RCS jets in wind tunnels is to determine the interaction effects of the thruster plumes on the flowfield and vehicle surface pressures. To our knowledge, the Apollo program did not test for these interaction effects. Depending on the jet characteristics and the local flowfield, the interaction effects can either augment or decrease the thruster performance. These interaction effects can be obtained experimentally by testing a model in a wind tunnel with the thruster on (operational) and off, and subtracting the two results. The thruster is usually modeled using a simulant gas, matching the mass and jet-to-freestream momentum ratio and plume geometry. For simplicity, the RCS thruster can be simulated with cold $\mathrm{N}_{2}$.

The NO PLIF method has been successfully applied in a variety of supersonic and hypersonic flow facilities. ${ }^{4,5,6,7,8,9,10,11,12}$ Also, PLIF of $I_{2}$ has previously been performed in a hypersonic wind tunnel at NASA Langley Research Center ${ }^{13}$ and elsewhere. However, this paper reports the first application of NO PLIF in a hypersonic wind tunnel at NASA Langley Research Center. PLIF flow visualization was chosen for measuring the RCS jet flowfield for several reasons. First, alternate imaging techniques are either difficult to implement or are not likely to provide images of good contrast in this flow. For example, while the commonly-used schlieren technique provided good visualizations of the forebody shock shape, it is not very sensitive in low pressure wake regions such as that probed in this experiment, as demonstrated below. Similarly, imaging the vehicle wake/jet region with Rayleigh scattering ${ }^{14}$ would produce poor-quality images owing to the low density there. Particle-based velocimetry techniques like particle image velocimetry ${ }^{15}$ could be used, but the presence of particles could affect the flowfield or could clog the small RCS jet orifice. PLIF is ideally suited for visualizing RCS jets. Nitric Oxide (NO) can be seeded directly into the $\mathrm{N}_{2}$ jet gas simulant; since NO has nearly the same molecular weight as $\mathrm{N}_{2}$, there is no question of particle or heavy molecule velocity slip or lag. However, NO is a toxic gas and must be appropriately handled. Though the quantities flowed in this experiment are relatively small, special safety precautions are taken when testing with NO. Unlike schlieren and Rayleigh scattering, whose sensitivity decreases with decreasing pressure, the NO PLIF signal intensity is approximately independent of pressure for a large range of low pressures. PLIF of the RCS jet yields strong signals with high contrast between the seeded jet fluid and the unseeded gas passing over the model. Another advantage of using PLIF for flow visualization is that it is relatively easy to perform velocimetry in hypersonic flows if a PLIF flow visualization is already being performed. ${ }^{16}$

\section{Experimental Description}

The experiment was performed in the 31-Inch Mach 10 Air wind tunnel at the NASA Langley Research Center. The test apparatus consisted of three main components: the test model, the wind tunnel facility and the optical visualization systems, which are detailed below.

\section{A. Model Geometry}

This model was designed and the experiment performed after U.S. President George W. Bush announced the Vision for Space Exploration in January 2004 but prior to the release of the CEV Exploration Systems Architecture Study (ESAS) in September 2005. ${ }^{1}$ During this time period, it was anticipated that the CEV would use a capsule-like entry vehicle, but the specific outer mold lines contours were not known. Ultimately, the ESAS team chose an axisymmetric capsule closely resembling, but larger than, Apollo. This is the same generic shape used in the present experiment. 

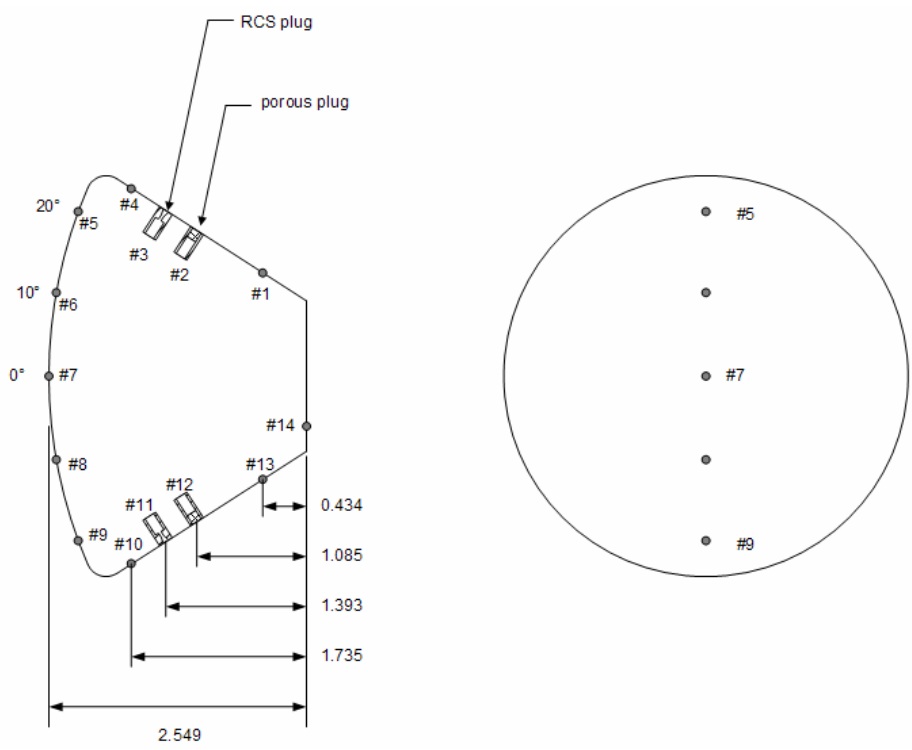

Figure 1. Capsule design: side view (left) and front view (right). Measurement units are inches.

\section{B. Planar laser-induced fluorescence (PLIF) Imaging System}

The PLIF system consists primarily of the laser system, beam forming optics and the detection system. The laser system has three main components: a pump laser, a tunable pulsed dye laser (Spectra Physics PDL-2), and a wavelength extender (Spectra Physics WEX). An injection-seeded Nd:YAG laser (Spectra Physics Pro-230-10) operating at $10 \mathrm{~Hz}$ pumps the PDL which contained a mixture of Rhodamine 590 and Rhodamine 610 laser dyes in a methanol solvent. The output of the dye laser and the residual infrared from the Nd:YAG are combined in a WEX containing both a doubling and a mixing crystal. The resulting output is tuned to a wavelength of $226.256 \mathrm{~nm}$, chosen to excite the strongly fluorescing spectral lines of $\mathrm{NO}$ near the $\mathrm{Q}_{1}$ branch head.

A monitoring gas cell system is used to ensure that the laser is tuned to the correct spectral line of NO. The gas cell contains a low-pressure mixture of $5 \% \mathrm{NO}$ in $\mathrm{N}_{2}$. A quartz window serves as a beam splitter and sends a small portion of the laser energy through windows on either side of the gas cell. A photomultiplier tube (PMT) monitors the fluorescence intensity through a third window at right angles to the path of the laser beam.

The components of this laser system are mounted within a twolevel, enclosable, portable cart. A photograph of this portable PLIF system is shown in Fig. 2 with the panels removed to show the internal components. When all of the panels are in place, a single monochromatic ultraviolet laser beam exits the cart, creating a relatively safe operating environment. Further details of the system can be found in Reference 17 .

For the experiments reported herein, this portable system was installed adjacent to the NASA Langley Research Center 31-Inch Mach 10 Air wind tunnel. A dedicated, adjustable scaffolding with attached mirrors and prisms directed the UV laser beam to the top of the wind tunnel test section. Optics then formed the beam into a $100 \mathrm{~mm}$ wide by $\sim 1 \mathrm{~mm}$ thick laser sheet, which was directed vertically downward through a window in the top of the test
The model design was based on the Apollo geometry from various reports of that time. The heat shield radius is 1.2 times the diameter, the corner radius is 0.05 times the diameter, and the backshell angle was 33 degrees. The model is attached to a sting at a $28^{\circ}$ angle, which was approximately the entry angle of attack of Apollo. Several pressure orifices (limited in number by the inside diameter of the sting) were placed on the model to measure model pressures but also to offer different seeding locations for the NO PLIF system. In addition to standard surface orifices, an RCS plug and a porous plug were included. The RCS plug incorporated a conical nozzle with an expansion ratio that provided an exit Mach number of 2.94. The porous plug consisted of a sintered metal plug 0.25-inch in diameter to diffuse the NO flow so as to not disturb the local flow. However, results obtained using this porous plug are not reported here.

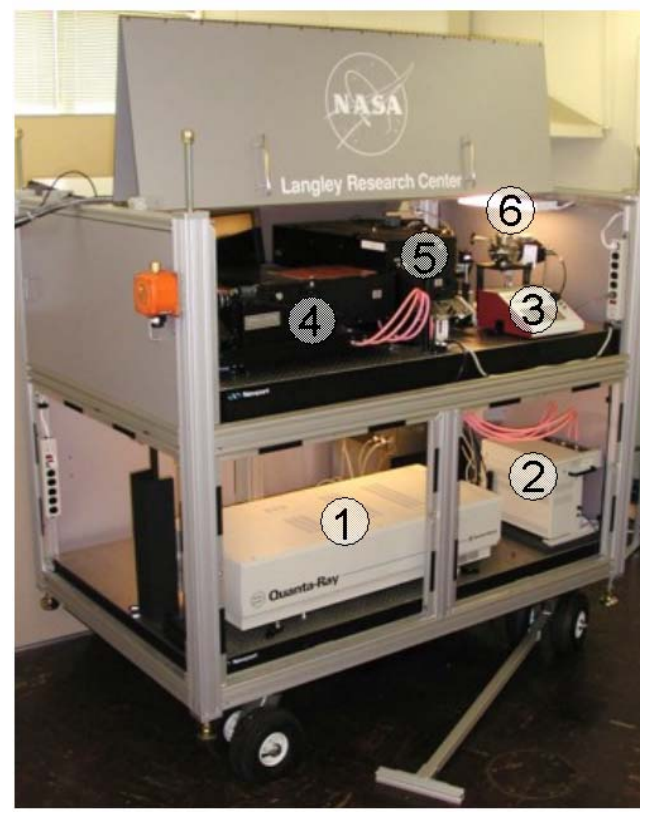

Figure 2. The portable PLIF system, shown with panels removed. Components include: (1) Nd:YAG laser; (2) dye circulators; (3) wavelength controller for the (4) pulsed dye laser; (5) wavelength extender; and (6) lowpressure monitoring gas cell. 
section. The section of scaffolding directly above the test section was mounted to a translation stage that could be remotely controlled so that the laser sheet could be swept spanwise through the flowfield during a tunnel run. This was used for alignment of the laser sheet and also for scanning the image plane through the flowfield to visualize three-dimensional flow structures. The resulting fluorescence from NO molecules in the flow was imaged onto a gated, intensified CCD camera at a viewing angle normal to the laser sheet. A 3-mm thick Schott glass UG5 filter was placed in front of the camera lens in order to attenuate scattered light at the laser's frequency. This was particularly important when the laser sheet impinged on the surface of the model potentially resulting in direct reflections towards the camera.

Flow visualization images were acquired at $10 \mathrm{~Hz}$ with a $1 \mu$ s camera gate and a spatial resolution of approximately 5 to 7 pixels/mm, varying from run to run as different camera views and laser sheet orientations were used. An image of a scale was obtained with the PLIF camera for the different camera placements so that the magnification could be determined in post-processing. The temporal and spatial resolution was more than sufficient to resolve flow structures of interest. Further details of the laser and camera system can be found in Reference 17.

Sets of 100 single-shot images were acquired for most measurements. Background images were also acquired on each day of testing. During the acquisition of these background images, the laser was firing but no gas was flowing through the RCS jet. Any nonzero intensity in these background images is due to either camera dark current or the laser scatter and room light not blocked by the filter in front of the camera lens. Background images were created from the average of 100 single-shot images in order to smooth out random shot-to-shot variations in background intensity in post processing.

\section{Schlieren}

A vertically-mounted lens schlieren system is used for flow visualization in the 31-Inch Mach 10 Air wind tunnel. The schlieren system consists of two $150-\mathrm{mm}$ diameter, $1000-\mathrm{mm}$ focal length achromat lenses, a point light source, a knife-edge and CCD video camera all mounted on an adjustable frame above and below the tunnel. High quality polished windows above and below the test section of the tunnel provide optical access.

The light source used is an Oriel type 6427 xenon arc lamp with a 3-mm arc length. An Oriel 68826 power supply is used to operate the flashlamp at 60 flashes per second with $800 \mathrm{~mJ}$ per flash. At these settings, the flash output has a duration of approximately 10 microseconds. The flashlamp is mounted in an Oriel model 60000 housing that uses a condenser lens to image the light onto a 1-mm diameter pinhole. The flashlamp assembly is located one focal length away from the first 150-mm diameter lens to collimate the light through the tunnel. Below the tunnel, the second 150-mm diameter lens captures the entire collimated beam and focuses the light onto a knifeedge, mounted on a three-axis positioner. Just below the knife-edge, a Hitachi KP-M1U monochrome CCD video camera is positioned to image the flow visualization during a test. Images are recorded on a PC with a frame grabber.

In the current configuration, the schlieren and PLIF systems cannot be used simultaneously. Schlieren was set up and used on repeated runs after the conclusion of the PLIF experiments. Due to the orientation of the schlieren system, the capsule model was rotated 90 degrees about the sting axis, to provide the schlieren images shown in this paper. All schlieren images were obtained with an angle of attack of the sting equal to zero (model angle of attack of 28 degrees).

\section{Wind Tunnel, Operating Conditions, Mass Flow Control and Data Acquisition Systems}

The 31-Inch Mach 10 Air wind tunnel is an electrically-heated blowdown facility located in Building 1251 at NASA Langley Research Center in Hampton, Virginia, USA. Reference 18 details this facility, a summary of which is provided here. As the name implies, the facility has a nominal Mach number of 10 and a 31-inch square test section. The tunnel uses heated, dried, and filtered air as the test gas. The air flows from the high pressure heater, through the settling chamber, three-dimensional contoured nozzle, test section, second minimum, aftercooler and into vacuum spheres pumped by a steam ejector and conventional vacuum pumps. The test section is "closed," as opposed to an "open jet" test section. Large windows form three walls (including top and bottom) of the test section with the fourth wall formed by the model injection system. This window arrangement has advantages in the present experiment because the laser sheet can be directed into the test section from the top window and fluorescence can be detected from the side. Also, the CCD camera can be placed very close to the test section windows, resulting in a working distance slightly larger than half of the test section width, allowing good-magnification (7 pixels $/ \mathrm{mm}$ ) PLIF images to be obtained without modification of the tunnel and without using exotic camera optics. Furthermore, the tunnel was already equipped with windows composed of UV-grade fused silica, providing $\sim 90 \%$ transmission at the $225 \mathrm{~nm}$ and higher wavelengths required for PLIF. 
Test durations of up to two minutes are possible in this facility. Tests could be performed approximately once per hour. The facility stagnation pressure $\mathrm{P}_{\mathrm{o}}$ can be varied from $350 \mathrm{psia}(2.41 \mathrm{MPa})$ to $1450 \mathrm{psia}(10.0 \mathrm{MPa})$ to simulate a range of Reynolds numbers. ${ }^{18}$ Two different stagnation pressures were used in this experiment: 350 psia (2.41 MPa) and 720 psia (4.96 MPa), corresponding to freestream unit Reynolds numbers of 0.5 and 1.0 million per foot, respectively. The test core size varies from about $12 \mathrm{in.} \times 12 \mathrm{in} .(0.30 \mathrm{~m} \mathrm{x} 0.30 \mathrm{~m})$ at the lowest stagnation pressure to $14 \mathrm{in}$. 14 in. $(0.36 \mathrm{~m} \mathrm{x} 0.36 \mathrm{~m})$ at the highest pressure. ${ }^{18}$ The nominal stagnation temperature was $1800^{\circ}$ Rankine $(1000 \mathrm{~K})$ for the experiment described herein. The freestream temperatures are estimated to be between $90^{\circ}$ and $95^{\circ}$ Rankine (50 and $53 \mathrm{~K}$ ), depending on chosen operating conditions. ${ }^{18}$ The freestream velocity is estimated to be about $4640 \mathrm{ft} / \mathrm{s}(1414 \mathrm{~m} / \mathrm{s}){ }^{18}$ The freestream pressure was estimated to be $0.0099 \mathrm{psia}$ (68 Pa) for the $\mathrm{P}_{\mathrm{o}}=350$ psia condition and to be 0.0187 psia $(129 \mathrm{~Pa})$ for the $\mathrm{P}_{\mathrm{o}}=720$ psia condition. ${ }^{18}$ Model surface pressures were determined using electronically scanned pressure (ESP) piezoresistive silicon sensors connected through 4 foot long tubes to the model. This length contributed to a delayed time response. The fore- and aft-body pressure orifices were 0.040 and 0.060 inches in diameter respectively. The 10 -inch water column (0.36 psi) ESP module was enclosed in the tunnel injection box and thus out of the airstream. The reference side of the module was held at a low vacuum pressure. At the lowest stagnation pressure conditions, ports on the aft-side of the model took nearly a minute to stabilize. Forebody pressures stabilized within a few seconds. Facility and model temperatures, pressures, angles of attack, etc. were recorded by a data acquisition system at a rate of $20 \mathrm{~Hz}$.

The 31-Inch Mach 10 Air wind tunnel facility is not equipped with a toxic gas cabinet for handling gas bottles containing nitric oxide (NO). Since this was a proof of concept study, a safe but inefficient method was used to supply NO. A small ( $\sim 0.5$ liter; $~ 17$ fluid oz) vessel was pressurized with a 95\% $\mathrm{N}_{2}$, 5\% NO mixture to $\sim 100$ psia $(\sim 700 \mathrm{kPa})$. This small volume and fill pressure was used to minimize the quantity of NO gas that would have been present in the room in the event of a leak. If a leak had developed, the NO concentration would not have exceeded the OSHA approved 8-hour time weighted average limit $(25 \mathrm{ppm})$, assuming that all of the gas mixed/diffused into a 3-foot (1-meter) radius around the leak. The gas bottle needed to be refilled after 3-5 tunnel runs. The NO/ $\mathrm{N}_{2}$ mixture flowed through mass flow controllers and then through the sting and into the model through stainless steel tubing embedded in the model. This tubing connected to the RCS jet nozzle. The mass flow controllers were made by Teledyne Hastings; the primary controller used in this experiment had a maximum flowrate of 1 standard liter per minute (SLPM) and an accuracy of $\pm(0.2 \%$ of full scale $+0.5 \%$ of reading), or about 0.005 SLPM for the conditions used. Nominal flowrates used in this experiment were 0.5 and 0.2 SLPM. The flowrates were recorded by the data acquisition system at $20 \mathrm{~Hz}$.

The normal sequence of operation was to begin flowing the NO/ $\mathrm{N}_{2}$ mix through the RCS jet, then to begin the tunnel flow and wait until both flows stabilized; then the data acquisition was started, the model was injected into the flow and the camera acquisition was started (sometimes more than once per run). A manual trigger indicated to the data acquisition system that the PLIF image acquisition had begun.

\section{Analysis Methods}

\section{A. PLIF Flow Visualization Image Processing}

Single-shot PLIF images were processed to subtract off background scattered light and camera dark current but the spatial variations in laser sheet intensity were not corrected. Both the background image and the single-shot images were smoothed with a filter (MATLAB ${ }^{\circledR}$,s 3 pixels x 3 pixels rotationally symmetric low-pass "fspecial()" Gaussian filter with a standard deviation of 1) prior to additional processing in order to reduce noise in the images. A background image was then subtracted from the filtered single-shot image. These images were then made into bitmap images or movies for display on the model using the Virtual Diagnostics Interface (ViDI) described below. Some flow visualization images were subsequently processed to determine the jet centerline, as described below, so that the trajectory and the flapping of the jet could be quantified.

\section{B. PLIF RCS Jet Trajectory Analysis}

PLIF images of the RCS jet were analyzed to determine the centerline of the jet. Sets of 100 individual visualization images were processed with custom-written MATLAB ${ }^{\circledR}$ software previously used for velocimetry. ${ }^{19,16}$ The images were first smoothed with MATLAB ${ }^{\circledR}$ s 5 pixel x 5 pixel Gaussian filter using a standard deviation of 2 pixels. Then each column in the image was fitted with a Gaussian function to determine the position of the jet within that column with sub-pixel accuracy. As the jet propagates downstream in the image it spreads out and dissipates. When the signal intensity dropped below a pre-set threshold value, the fitting process was terminated to avoid fitting to noise. The measured trajectories were then converted from pixels into mm using the imaging system magnification, which was measured by imaging a scale. The magnification varied between 5 and 7 pixels $/ \mathrm{mm}$ 
between the different images analyzed. For each column in the image, an average and a standard deviation of the jet centerline was determined from the 100 images. These averaged jet centerlines are then graphed with the origin of the graph located at the center of where the RCS jet exits the capsule.

\section{Virtual Diagnostics Interface (ViDI)}

The Virtual Diagnostics Interface (ViDI) ${ }^{20}$ is a software package developed at NASA Langley Research Center that provides unified data handling and interactive 3D display of experimental and theoretical data. Currently this technology is applied to three main areas: 1) pre-test planning and optimization; 2) analysis and comparative evaluation of experimental and computational data in near real time or in post-processing; and 3) establishment of a central hub to source, store and retrieve experimental results. ViDI is a combination of custom applications and the 3D commercial software Autodesk ${ }^{\circledR} 3 \mathrm{ds} \mathrm{Max}^{\circledR}{ }^{21}$

For this experiment, ViDI was used for post-test visualization of PLIF and schlieren data. A CAD (Computer Aided Design) file for the Apollo capsule was imported into the virtual environment along with the PLIF and schlieren images. The images were then scaled and placed over the Apollo capsule. To create the final output, a virtual camera was placed in the scene, and high resolution bitmaps were rendered. In addition, a sequential series of files containing PLIF imagery was imported to create animations of time-varying data.

\section{Conventional Data Systems}

The surface pressure data were averaged over the last 2 seconds of each camera sequence (see prior description of tunnel/data acquisition procedure). By this time, the pressures had reached a limiting value (i.e. were settled). The pressures were then converted to pressure coefficient $\left[\mathrm{C}_{\mathrm{p}}=\left(\mathrm{P}-\mathrm{P}_{\infty}\right) / \mathrm{q}_{\infty}\right]$, where $\mathrm{P}_{\infty}$ and $\mathrm{q}_{\infty}$ are the freestream static and dynamic pressures, respectively. The 2-sigma uncertainty in $C_{p}$ was estimated as \pm 0.002 , both from a propagation-of-errors method and from within-run and run-to-run repeatability.

\section{Results}

Three parameters were varied in the experiment: mass flow rate of the RCS jet, stagnation pressure of the wind tunnel, and model angle of attack. Also two different laser sheet orientations were used. These data sets are discussed individually in the sections below and then holistically in the Discussion section below.

\section{A. PLIF and Schlieren Flow Visualizations}

\section{Qualitative Interpretation}

Figure 3 shows a ViDI rendering of the capsule model with a single-shot false-color NO PLIF image overlaid. For this measurement, the laser sheet entered the flowfield from the top, was oriented parallel to the sting and was on the model centerline. The camera was placed at a right angle to the laser sheet. Unless otherwise specified, all visualizations shown in this paper were obtained using this configuration. In the ViDI rendering, the virtual camera was oriented from about a $45^{\circ}$ angle and above the model to better show the details of the model and the location of the laser sheet.

The rendering shows that the RCS jet is emitted from its nozzle normal to the model's aft surface. The jet expands into a classic barrel shock pattern in the near field, indicating that it is highly underexpanded. Downstream of this structure, the jet bends dramatically back towards the model centerline. The jet curves in this location because it is approaching the shear layer that separates the low-speed gas in the capsule's separated wake from

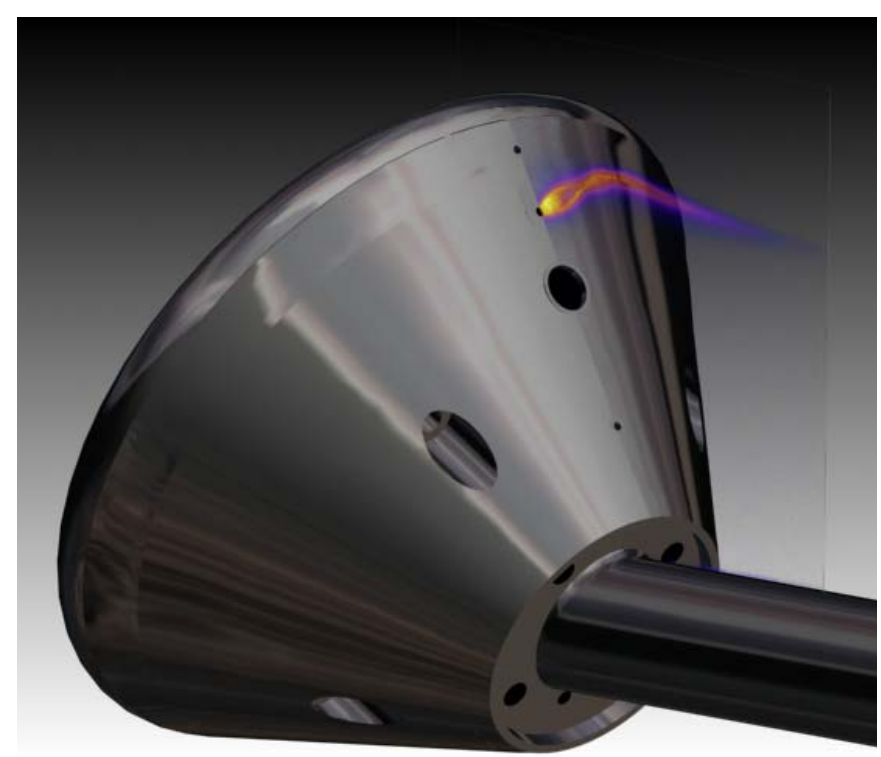

Figure 3: ViDI rendering of the capsule model with PLIF visualization of the RCS jet. 
the high-speed gas coming from the capsule’s forebody. ${ }^{\ddagger}$ As the jet propagates further downstream, it is observed to decrease in intensity. There are two main causes for this decrease in PLIF intensity. As will be shown below, the jet is forming into a horseshoe shape. Thus, much of the jet gas is being transported away from the measurement plane. Since the fluorescence intensity is proportion to the NO concentration, the signal decreases. The second effect is that $\mathrm{O}_{2}$ from the tunnel gas is mixing with the jet gas on a molecular scale as the jet propagates. Since $\mathrm{O}_{2}$ quenches the fluorescence of NO, the fluorescence signal decreases as more mixing occurs.
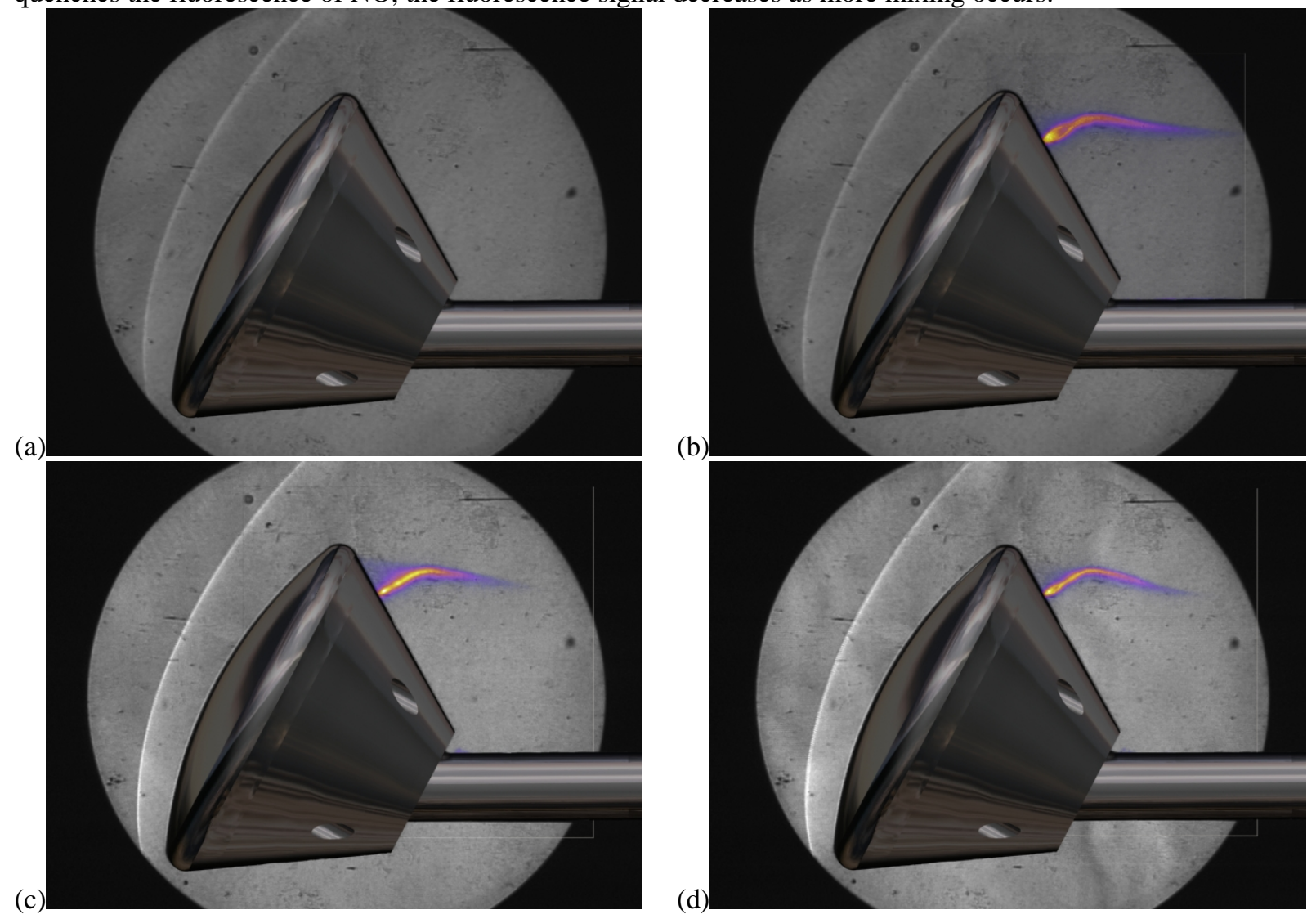

Figure 4. PLIF and Schlieren visualizations for (a) $\dot{m}=0.5$ SLPM, $\mathbf{P}_{\mathbf{0}}=350$ psia (schlieren only), (b) $\dot{m}=0.5$ SLPM, $\mathbf{P}_{0}$ $=350$ psia, (c) $\dot{m}=0.2$ SLPM, $\mathbf{P}_{\mathbf{0}}=720$ psia, (d) $\dot{m}=0.5$ SLPM, $\mathbf{P}_{\mathbf{0}}=720$ psia.

Figure 4 shows comparisons between PLIF and Schlieren for various operating conditions. The schlieren image in Figure 4(a) clearly identifies the bow shock on the model. This figure shows an average of 20 single-shot schlieren images so that shot-to-shot variations are averaged out. The black marks randomly located around the image are caused by dirt and scratches on the optics in both the schlieren system and tunnel windows. For these schlieren measurements, the mass flow rate of NO from the RCS jet was 0.5 SLPM, its maximum value used in this study. However, the jet cannot be seen in the schlieren image. It is possible that the sensitivity of the schlieren system could have been improved to visualize the jet, but clearly this flow visualization method does not easily visualize the jet. Another observation from the schlieren images was that the images obtained with the RCS jet flowing were indistinguishable from those with no RCS jet. That is, the forebody shockwave did not appear to change shape with fluid injection at this location. Figures 4(c) and 4(d) show single-shot schlieren images obtained at different times. Note that these single images have sharper shock waves than in the averaged image of Fig. 4(a). Also, note the slightly different curvatures in the bow shocks in the two figures. This is typical of the observed small degree of bow shock unsteadiness observed in the experiment.

Figure 4(b) shows the same PLIF visualization of the RCS jet shown in Figure 3, but from a different virtual camera orientation, and overlaid with schlieren. Figure 4(d) shows a PLIF image obtained with the same RCS jet mass flow rate but with the tunnel stagnation pressure about $2 x$ higher. Consequently, the underexpanded barrel

\footnotetext{
抹 A series of separate PLIF visualizations, not reported here, but obtained at the same time as these substantiates this claim.
} 


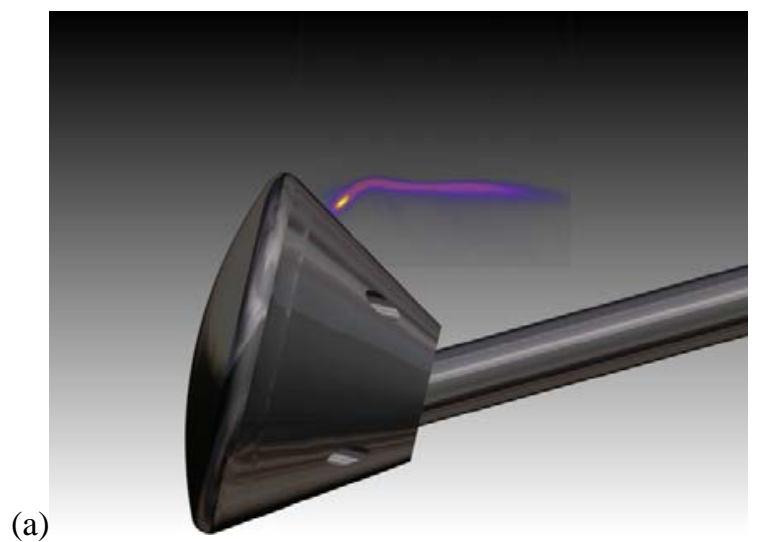

(a)

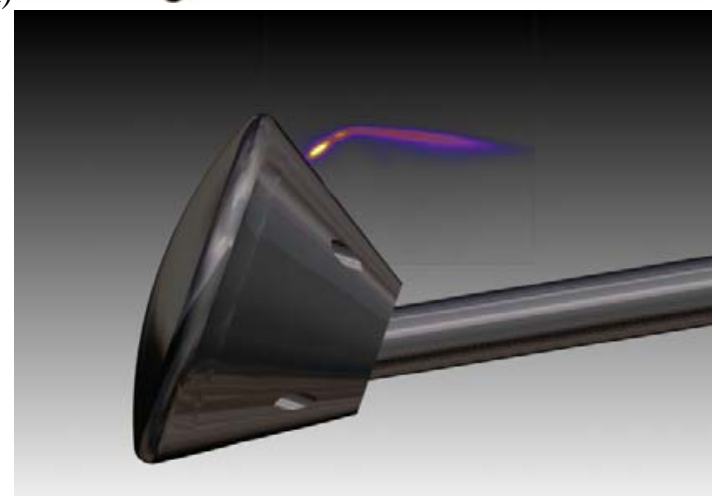

(b)

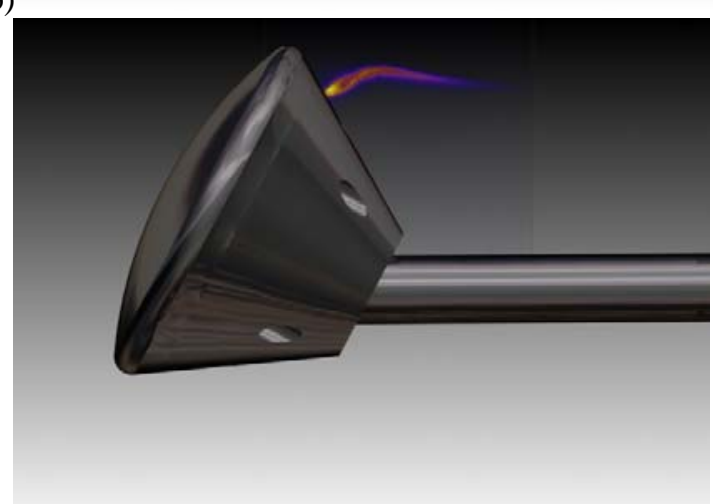

(c)

Figure 5. Angle of attack variation during a tunnel run. The model angle of attack was $13^{\circ}(\mathrm{a}), 20^{\circ}$ (b) and $28^{\circ}$ (c). The $\mathbf{P}_{\mathbf{o}}=350$ psia and $\dot{m}=0.5$ SLPM.

Many flowfields are highly three dimensional, including this one. Imaging along the centerline of the model does not describe the flowfield fully. To systematically obtain measurements at other locations in this flow, the laser sheet was swept through the flow during some runs. The laser was still impinging on the model from above and the width of the laser sheet was still parallel to the axis of the sting and the tunnel. However, the sheet no longer coincided with the centerline of the model. It was placed $10 \mathrm{~mm}$ to the far side of the jet centerline (from the camera's perspective) and was swept towards the camera at the rate of $2 \mathrm{~mm} / \mathrm{s}$. In 10 seconds, the entire three dimensional flowfield was sampled. In post-processing, we converted these one hundred 512 x 512 pixel images into five hundred and twelve 100 x 512 pixel composite images. Figure 6 shows 8 of these 100 x 512 pixel images overlaid on the model. These images show the evolution of the shape of the jet as it propagates downstream. The shock is smaller, though the general behavior of the flow is similar. In Figure 4(c), the conditions are the same as Fig. 4(d), except with a reduced RCS jet flowrate (0.2 SLPM). Note the even smaller barrel shock pattern (barely distinguishable) and also note that the jet does not appear to penetrate as far into the shear layer. To summarize, the nearfield shape of the RCS jet plume depends on both the jet gas flowrate and the tunnel stagnation pressure, both of which affect the pressure ratio across the RCS jet nozzle.

The angle of attack of the model was varied during one run. The angle of attack of the sting was varied from $-15^{\circ}$ to $0^{\circ}$ during a 10 -second run. This corresponds to the model angle of attack varying from $13^{\circ}$ to $28^{\circ}$. A handpressed trigger was used to synchronize the PLIF acquisition to the angle of attack measurement in the data acquisition system. The accuracy in this manual timing is estimated to be about 0.5 seconds, which corresponds to about $1^{\circ}$ error in matching sting angle with PLIF image. ViDI rendered these images into a movie showing the change in the shape of the jet during this sweep. Three still frames from of this measurement are shown in Figure 5. The shape and trajectory of the jet changes as the angle of attack varies.

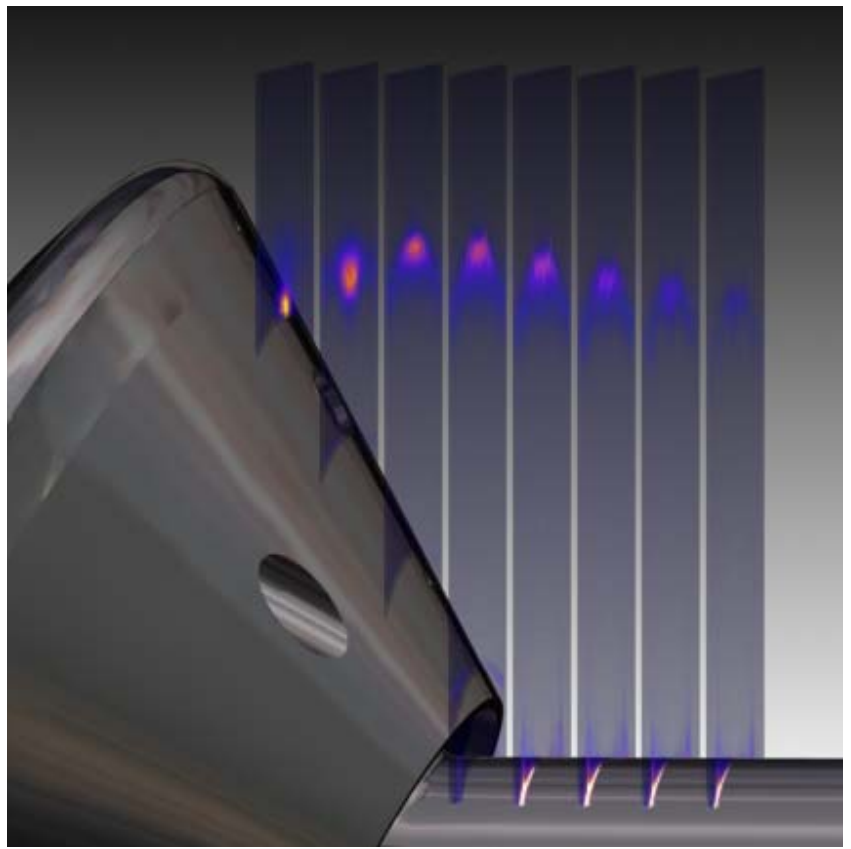

Figure 6: ViDI rendering of eight equally spaced planes of data generated by sweeping the laser sheet, which was aligned parallel to the sting, spanwise through the flow. The cross sections shown above were computed with image processing. 
jet is observed to evolve from round in the near field to horseshoe shaped in the far field. This is typical for a jet in a crossflow. No smoothing has been applied to these images.

To obtain a direct image of the cross section of the RCS jet, it was desired to orient the laser sheet perpendicular to the tunnel axis and to sweep the laser sheet along the tunnel axis while visualizing the fluorescence from a downstream location. This was not possible for practical reasons including the fact that there is no optical port downstream. However, a compromise was obtained by orienting the laser sheet at a 45 degree angle with respect to the tunnel centerline and orienting the camera at a right angle to this sheet. As the laser sheet swept in the horizontal direction, different jet cross sections were intersected and visualized. Figure 7 shows some renderings obtained using this method. Again, the jet is observed to evolve from circular (elliptical in this view since the laser sheet is at 45 degrees with respect to the jet) to horseshoe shaped.

\section{Quantitative Interpretation: Measured Jet Trajectories}

These flow visualization images provided quantitative information about the trajectory of the RCS jet for different operating conditions. The trajectories of the individual jets were determined using the method described in section III B. Figure 8 shows the measured trajectory for one operating condition. Note that the vertical and horizontal scales differ - the vertical scale has been expanded. The figure shows the averaged centerline position of the jet. The jet trajectory is approximately straight and perpendicular to the model surface in the near field, probably because it is propagating into a relatively quiescent (low speed) separated flow region aft of the model. The jet then approaches the aforementioned shear layer where it turns.

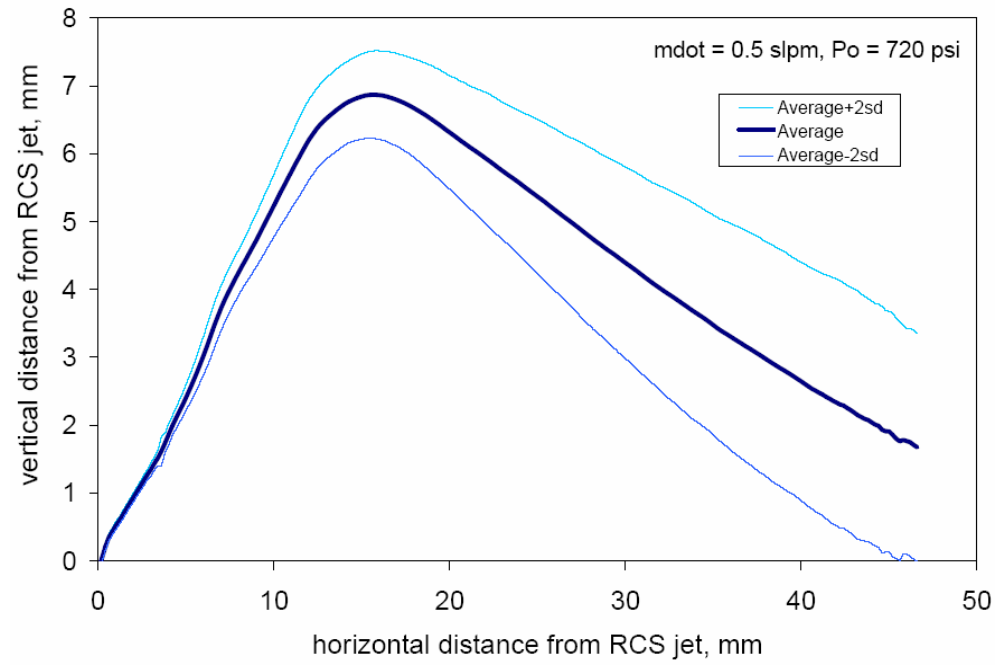

Figure 8: Averaged jet centerline trajectory (thick curve) and jet centerline range caused by flapping. The flapping range is shown by the thin curves which are the average trajectory \pm 2 standard deviations (sd).

Figure 8 also shows the range over which jet centerline fluctuates. A range of \pm 2 standard deviations was plotted to indicate the range of jet flapping during the 10 second measurement. The jet flapping increases as the jet propagates downstream from a negligible standard deviation of $0.03 \mathrm{~mm}$ at injection to a standard deviation $\sim 0.9 \mathrm{~mm}$ at the maximum. The maximum standard deviation $(0.9 \mathrm{~mm})$ is comparable to, but smaller than the jet plume diameter $(\sim 1.5-4 \mathrm{~mm}$, measured full width at half maximum). For comparison, the RCS nozzle exit diameter was $1.52 \mathrm{~mm}$ (0.060-inch). 
For the two runs in which 100 single measurements were obtained ( $\dot{m}=0.2$ and $\dot{m}=0.5$ SLPM for $\mathrm{P}_{\mathrm{o}}=720$ psia), the magnitude of the flapping was very similar: the maximum standard deviation was $\sim 0.9 \mathrm{~mm}$ for both runs.

Figure 9 compares averaged jet trajectories for three different experimental conditions. A gap in the data is present in the region of the barrel shock for the 0.5 SLPM, $\mathrm{P}_{\mathrm{o}}=350$ psia run. The Gaussian fit algorithm could not fit the data in this region because the columns of data contained two peaks in intensity. This same effect may also be influencing the fitted trajectory in the near field $(<8 \mathrm{~mm}$ horizontal distance) of the other two cases. Until the jet reaches the shear layer, the three different jets share approximately the same linear trajectory. The 0.5 SLPM jets have very similar trajectories despite issuing into gas that has a different pressure by a factor of two. As

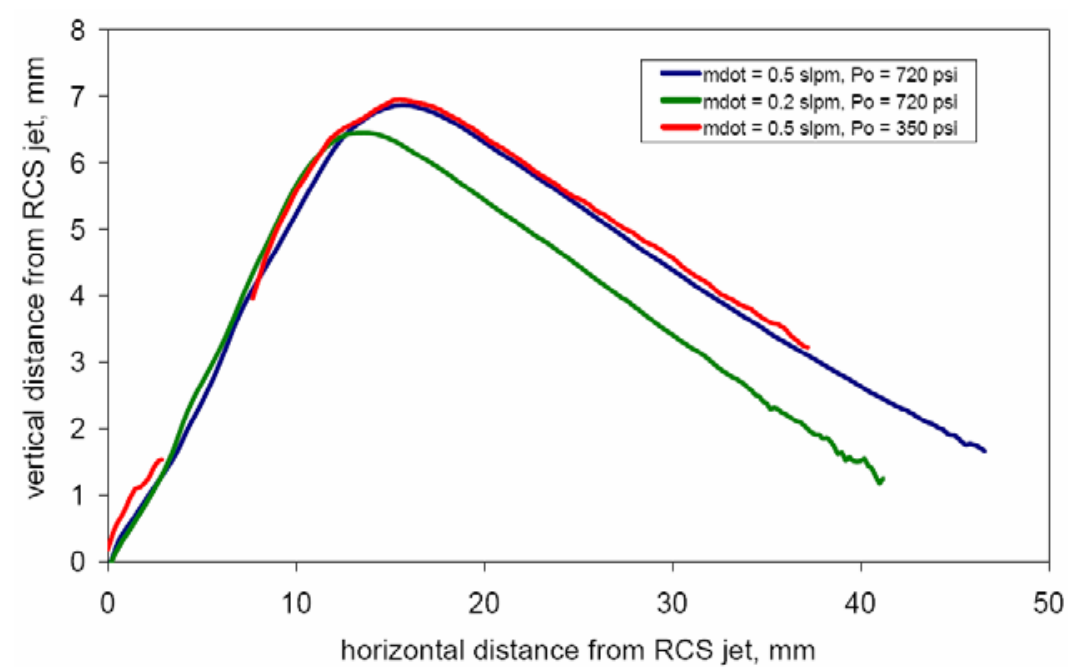

Figure 9: Comparison of averaged jet trajectories for different experimental conditions. mentioned above, the size of the barrel shock differs in the two flow visualizations at the two different tunnel stagnation pressures. Since the flowfields are visibly different, it was not anticipated that the centerline trajectories would be so similar. Further testing is required to determine whether this is a general trend or a coincidence. Comparing the 0.2 SLPM and 0.5 SLPM curves indicates that the jet having the higher flow rate (more momentum) penetrated deeper into the shear layer before turning, as expected.

The data from Figure 8 has been superimposed on a ViDI rendering of the model in Figure 10. This visualizes the jet trajectory in real spatial coordinates and also allows a double-check of the data in comparison with raw images.

(a)
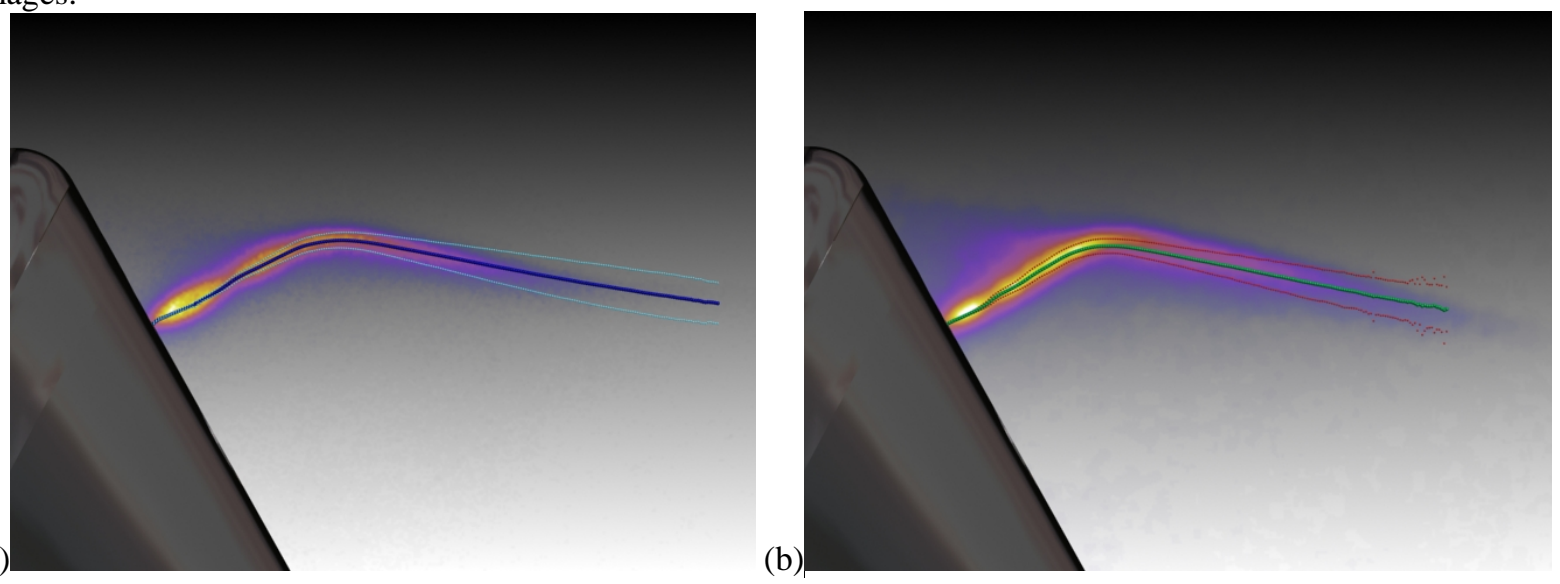

Figure 10. ViDI rendering of the average and average \pm 2 standard deviation of the measured jet centerlines (from Figs. 8 and 9) overlaid on typical single-shot PLIF images for (a) $\dot{m}=0.5$ SLPM, $P_{0}=720$ psia and (b) $\dot{m}=0.2$ SLPM, $P_{0}=720$ psia.

\section{B. Conventional Measurements}

The surface pressure data are presented in Figure 11 for a model angle of attack of $28^{\circ}, \mathrm{P}_{\mathrm{o}}=720$ psia, corresponding to a unit Reynolds number of 1.06 million, and mass flow rate of $0.5 \mathrm{slpm}$. On the horizontal axis, the distance, $s$, along the model has been normalized by the model radius, $R_{n}$ which is equal to $4.8 \mathrm{in}$. The RCS jet exhausted from port $3\left(s / R_{n}=0.594\right)$. No significant change in the surface pressures are noted when the RCS jet is operated at 0.5 SLPM or lower. Figure 12 presents the same data but with an expanded scale on the vertical axis. It 
seems that the surface pressures are lower with the RCS jet active for the two ports surrounding the RCS port, but the difference is within the quoted uncertainty level. Data at lower mass flow rates gave similar results.

It should be noted that the RCS jet was operated at low mass flow rates in the present experiment which was aimed at demonstrating the NO PLIF measurement technique. Proper simulation of flight hardware may require much larger flow rates and thus create greater interaction. These data at low flow rates however indicate that it may be possible to seed NO into the flow for wake visualization studies using PLIF without altering the flow under investigation.

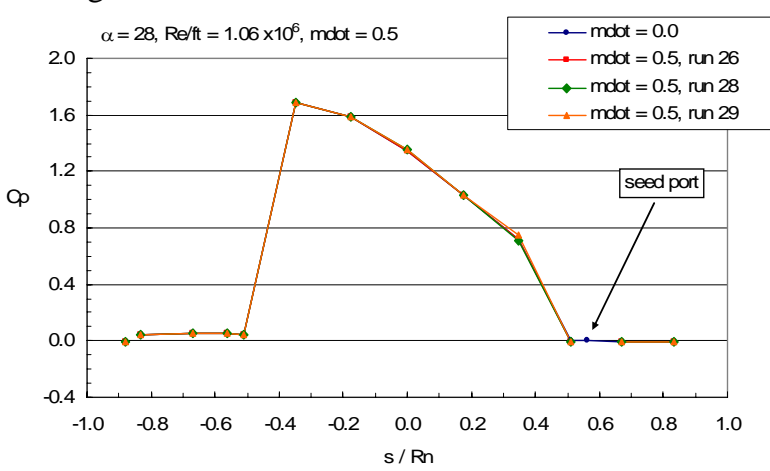

Figure 11. Pressure coefficient distribution on the model for tunnel runs with $P_{0}=720$ psia and varying $\dot{m}$.

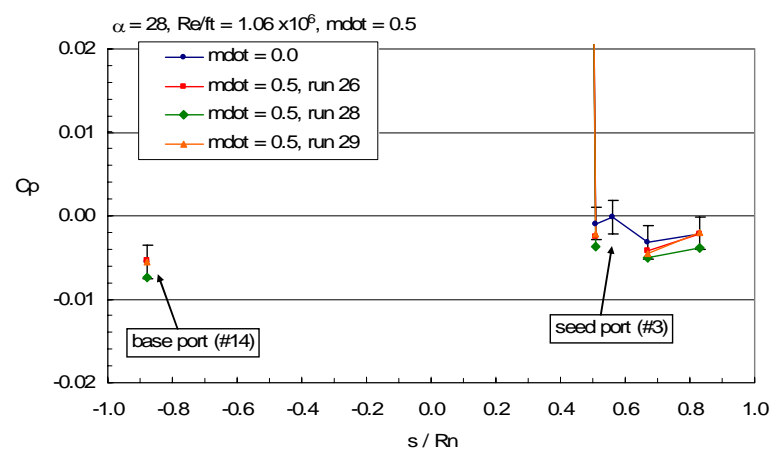

Figure 12. Expanded scale of model pressure distribution, emphasizing aft-side orifices. Error bars shown are \pm two standard deviations and are the same for each point, though they are only shown plotted on one run.

\section{Discussion}

The results show that the NO PLIF method can visualize RCS jet flows in hypersonic wind tunnels. Using image processing including the ViDI visualizations, both qualitative and quantitative information can be obtained. This information should prove useful for validating computational predictions of these flows. However, there were several lessons learned from this experiment. This section aims at identifying these lessons as well as suggesting solutions for problems experienced during the experiment.

The most important improvement that should be made to the system is to acquire images of a card with a regular pattern of dots each time the camera is moved or the lens is adjusted. The dot card should be placed in the plane of the laser sheet. The dot card has several uses. First, the spacing of the dots is carefully controlled so that the images can be de-warped, thereby correcting for lens distortions. Second, the magnification of the image can be accurately determined. Third, the dot card can help indicate where the images should be located relative to the model in the ViDI renderings. This is particularly important in the case where the laser is swept through the flowfield. In the present experiment, dot card images were not acquired. Instead, images of a ruler were obtained for most runs to determine the image magnification. However, lens distortions may have caused the image magnification to vary around the image. This could not be accounted for in the present experiment. In some cases the images were scaled by up to $5 \%$ to match to the rendered model. This image scaling could have been performed more systematically had a dot-card image been acquired. Similarly, in several of the experiments (see for example, Fig. 7), it was not possible to determine exactly where the image plane was during ViDI processing. Assumptions were required and the images did not line up exactly with the model, as seen in the top image of Fig. 7. Dot card images will be acquired in future experiments. Prior to performing complicated experiments like that shown in Fig. 7, test runs should be performed in a carefully controlled laboratory experiment simulating a wind tunnel. This would allow systematic methods to be developed that would avoid errors in ViDI renderings.

Another problem that occurred in post-processing the data was caused by using a manual trigger to document the start of a camera acquisition in the data file. This process has an uncertainty of about a half second. During a dynamic event such as an angle of attack sweep, a half second of timing uncertainty can result in a 1 degree angle of attack uncertainty - that is, it is uncertain which image goes with which angle of attack measurement in the data file. This problem has since been resolved by using an electronic pulse (instead of a manual one) generated by the camera controller.

The accuracy of the jet trajectory measurement has not yet been carefully assessed. Clearly, the precision of the technique is sufficient to resolve flapping of these jets, at least in the far field. The fitting algorithm is based on the one used in Reference 19. These researchers found that when the signal-to-noise ratio was high, the fitting algorithm was accurate to $1 / 10$ of a pixel. In the present experiment, $1 / 10$ of a pixel corresponds to $\sim 0.02 \mathrm{~mm}$ 
(0.0008 in.). This is a reasonable estimate of the precision of the measurement in regions of the flow with high signal-to-noise ratio. However, lens distortions have not been corrected, so the accuracy of the measurement could be affected by such distortions. Careful laboratory experiments could quantify errors in the method, such as those caused by lens distortions. The lens distortions could be removed with image processing in future experiments if dot-card images are obtained prior to or after performing the experiments. Care was taken to align the camera so that the rows of the CCD were parallel to the sting. Typically, the camera rows were parallel to the sting to a fraction of a degree. This was not a significant source of error in the experiments. The spatial resolution of the jet trajectory measurement was limited by the $5 \times 5$ pixel Gaussian smoothing algorithm, using a standard deviation of 2 pixels in MATLAB ${ }^{\circledR}$. This process degrades the effective spatial resolution of the measurement to about $1 \mathrm{~mm}$ (that is, the measured centerline is averaged over a region of about $1 \mathrm{~mm}$, though the position of this centerline could be determined to within $0.02 \mathrm{~mm}$ ). In retrospect, the signal-to-noise ratio was good enough in the present data set that a much smaller filter could have been applied, thereby improving the spatial resolution of the measurement by perhaps a factor of 4 . Another improvement to the software will be required to fit regions of the flow where the column of data does not have a Gaussian shape, such as in the barrel shock region of the jet. Finally, when the jet trajectory is not aligned perpendicular to the columns of the camera, a bias exists in the jet trajectory measurement. This is particularly a concern in the near field of the jet, where the jet is rapidly changing shape, for example in the $\dot{m}=0.2, \mathrm{P}_{\mathrm{o}}=350$ psia case shown in Fig. 4(b). Near the nozzle exit, the bottom of a given column corresponds to a location in the jet that is much closer to the nozzle exit than the top of the column. This has a tendency to bias the measured centerline data. It may instead be desirable to develop an algorithm that analyzes cross sections of the jet that are perpendicular to the jet. Nonetheless, when comparing with theoretical predictions of the jet trajectory, as long as the same method is used to find the theoretical jet centerline, the comparison should be valid.

In summary, the following observations and recommendations are noted for future PLIF tests of RCS Jets:

- For each adjustment of the CCD camera, images of a dot card should be acquired-preferably at several known locations in the flowfield to be probed.

- An experiment should be performed in a controlled laboratory environment simulating a wind tunnel in order to develop standard practices for dot-card image acquisition that will allow PLIF images to be correctly placed in ViDI renderings. This is particularly important if the laser sheet will be swept through a flowfield.

- The manual trigger for the data acquisition system indicating the start of a PLIF image acquisition should be replaced by a more accurate automatic trigger generated from the camera hardware.

- Jet trajectory software will need to be improved in a variety of ways-particularly if highly threedimensional jet trajectories are to be monitored.

- The combination of ViDI, schlieren, and PLIF can be useful for identifying differences between asdesigned and as-built model geometries.

\section{Conclusion}

The planar laser-induced fluorescence technique has been applied to study a RCS jet on a capsule entry vehicle similar to Apollo and the proposed CEV. PLIF was used to visualize the jet, to determine the centerline trajectory of the jet, and to determine the shape of the jet downstream of injection. These measurements were complemented by conventional pressure instrumentation. The experiment demonstrates the ability of PLIF to study RCS jets while providing a data set that can be compared to CFD simulations of the flowfield. Improved confidence in CFD simulations should result in lower uncertainties in the operation of the RCS jets during flight.

\section{Acknowledgments}

We wish to acknowledge the contribution to this project from the NASA Langley Research Center 31-Inch Mach 10 Air tunnel technicians and engineers, including Anthony Robbins, Kevin Hollingsworth, Rhonda Murphy, Henry Fitzgerald, Johnny Ellis and Paul Tucker. Also we acknowledge the contribution of Vince LeBoffe of NASA Langley Research Center for completing the model design. Also we acknowledge Rich Schwartz from Swales Aerospace, Hampton VA, for useful advice during the ViDI analysis.

\section{References}

\footnotetext{
${ }^{1}$ See http://www.nasa.gov/mission_pages/exploration/spacecraft/cev.html and http://www.nasa.gov/pdf/133654main_ESAS_charts.pdf.

${ }^{2}$ F. Sietzen Jr., "From Mercury to CEV: Space Capsules Reemerge,” Aerospace America, p. 26-33, Feb (2005).
} 


\footnotetext{
${ }^{3}$ C. A. Vaughan, “Apollo Reaction Control Systems,” AIAA Paper Number 68-566 (1968).

${ }^{4}$ Paul PH, Lee MP, Hanson RK, Molecular velocity imagining of supersonic flows using pulsed planar laser-induced fluorescence of NO, Opt. Lett., Vol. 14, 417-419 (1989).

${ }^{5}$ McMillin BK, Palmer JL and Hanson RK, "Temporally resolved, two-line fluorescence imaging of NO in a transverse jet in a supersonic cross-flow,” Appl. Opt., 32, 7532 (1993).

${ }^{6}$ W. L. Roberts, M. G. Allen, R. P. Howard, G. J. Wilson, R. Trucco, "Measurement and Prediction of Nitric Oxide Concentration in the HYPULSE Expansion Tube Facility,” AIAA 94-2644 (1994).

${ }^{7}$ W.M. Ruyten, M.S. Smith and L.L. Price, "Status of Laser-Induced Fluorescence and Planar Laser Induced Fluorescence Measurements in the AEDC HEAT-H2 Arc Heater Facility” AIAA 95-2038 (1995).

${ }^{8}$ W. M. Ruyten "Comparison of calculated and measured temperature fields in the AEDC Impulse Facility" AIAA-19962237 (1996).

9 Houwing AFP, Palmer JL, Thurber MC, Wehe SD, Hanson RK, Boyce RR, Comparison of planar fluorescence measurements and computational modeling of a shock layer flow, AIAA Journal, Vol. 34 (3), $470-477$ (1996).

${ }^{10}$ W. H. Beck, G. Eitelberg, O. Trinks, and M. Wollenhaupt, “Testing methodologies in the DLR High Enthalpy Shock Tunnel HEG,” AIAA-1998-2770 (1998).

${ }^{11}$ J. S. Fox, A. F. P. Houwing, P. M. Danehy, M. J. Gaston, N. R. Mudford, S. L. Gai, "Mole-Fraction-Sensitive Imaging of Hypermixing Shear Layers," Journal of Propulsion and Power, Journal of Propulsion and Power, 17(2), p. 284-292, (2001).

${ }^{12}$ P. C. Palma, P. M. Danehy, A. F. P. Houwing, "Fluorescence Imaging of Rotational and Vibrational Temperature in a Shock Tunnel Nozzle Flow," AIAA Journal, Vol. 41, no. 9, Sept. p. 1722-1732 (2003).

${ }^{13}$ R. J. Exton, R. J. Balla, B. Shirinzadeh, M. E. Hillard, and G. J. Brauckmann, "Wake imaging in supersonic facilities using the iodine Cordes bands," AIAA-1998-2697.

${ }^{14}$ Shirinzadeh, B., R.J. Balla, and M.E. Hillard, "Rayleigh scattering measurements in supersonic facilities," AIAA-96-2187, 19th AIAA Advanced Measurement and Ground Testing Technology Conference, New Orleans, LA, June 1996.

${ }^{15}$ Goyne CP, McDaniel JC, Krauss RH and Day SW, "Velocity Measurement in a Dual-mode Supersonic Combustor using Particle Image Velocimetry," AIAA/NAL-NASDA-ISAS 10th International Space Planes and Hypersonic Systems and Technologies Conference, AIAA 2001-1761, April 2001.

${ }^{16}$ P. M. Danehy, S. O’Byrne, A.F.P. Houwing, J.S. Fox, D.R. Smith "Flow-tagging velocimetry for hypersonic flows using fluorescence of nitric oxide,” AIAA Journal v. 41, n. 2, p. 263-271 (2003).

${ }^{17}$ J. A. Wilkes, D. W. Alderfer, S. B. Jones, and P. M. Danehy, "Portable Fluorescence Imaging System for Hypersonic Flow Facilities,” JANNAF Interagency Propulsion Committee Meeting, Colorado Springs, Colorado, December 2003.

${ }^{18}$ J. R. Micol "Langley Aerothermodynamic Facilities Complex: Enhancements and Testing Capabilities," AIAA Paper 980147, 36th AIAA Aerospace Sciences Meeting \& Exhibit, January 12-15, Reno, NV (1998).

${ }^{19}$ R B Miles, J Grinstead, R H Kohl and G Diskin "The RELIEF flow tagging technique and its application in engine testing facilities and for helium-air mixing studies,” Meas. Sci. Technol. v. 11, p. 1272-1281 (2000).

${ }^{20}$ Schwartz, R.J., "ViDI: Virtual Diagnostics Interface Volume 1-The Future of Wind Tunnel Testing" Contractor Report NASA/CR-2003-212667, December 2003

${ }^{21}$ http://www.autodesk.com/
} 